\title{
Cucurbitacin I inhibits cell migration and invasion and enhances chemosensitivity in colon cancer
}

\author{
JUNMIN SONG ${ }^{1}$, HONGXIANG LIU ${ }^{1}$, ZHEN LI $^{1}$, CHAO YANG $^{1}$ and CHAOJIE WANG ${ }^{2}$ \\ ${ }^{1}$ Department of General Surgery, First Affiliated Hospital of Zhengzhou University, Zhengzhou 450052; \\ ${ }^{2}$ Department of Oncology, Henan Provincial People's Hospital, Zhengzhou 450003, P.R. China
}

Received November 7, 2014; Accepted December 31, 2014

DOI: 10.3892/or.2015.3749

\begin{abstract}
Colorectal cancers are the third most common types of cancers worldwide. Surgical resection is unable to eliminate tumors completely due to metastasis. A demand for new chemotherapeutic tools exists. In the present study, we examined the chemopreventive potential of cucurbitacin I, a natural component extracted from plants of the Cucurbitaceae family, in the colon cancer cell line COLO205. We hypothesized that cucurbitacin I would prevent colon cancer cell migration and invasion, and sensitize colon cancer cells to chemotherapy. Our data demonstrated that exposure of the COLO205 cells to cucurbitacin I significantly decreased cell viability. Furthermore our data demonstrated for the first time that in the COLO205 cells, cucurbitacin I could suppress the cell migration and invasion, and harbor chemosensitization activity against colon cancer. The anticancer activity of cucurbitacin I was accomplished by downregulating p-STAT3 and MMP-9 expression. Collectively, our results suggest that cucurbitacin I may be a potent adjuvant chemotherapeutic agent for colon cancer with anti-migration, anti-invasion and chemosensitizing activities.
\end{abstract}

\section{Introduction}

Colorectal cancers are the third most common types of cancers worldwide (1-3). Surgical resection is unable to eliminate tumors completely since metastasis occurs at the time of diagnosis in approximately three-fifths of the patients. The 5-year survival rate is also very low due to metastasis (4-6). At present, there is no effective adjuvant chemotherapy, and rationally designed new adjuvant therapeutic tools need to be developed to manage the metastatic process in colorectal cancer patients. In the past several decades, a large number of substances derived from plants has been studied in antitumor

Correspondence to: Dr Junmin Song, Department of General Sugery, First Affiliated Hospital of Zhengzhou University, 1 East Jianshe Road, Zhengzhou 450052, P.R. China

E-mail: songjunmin99@163.com

Key words: cucurbitacin I, cell migration, cell invasion, chemosensitivity, 5-FU research fields and many have proven to exhibit chemopreventive properties (7-10), which could be used as adjuvant chemotherapy.

Cucurbitacin I, also known as elatericin B, is a member of a family of natural occurring compounds with potent antitumor activity in many human cancers, including glioblastoma, adenocarcinoma of the lung and breast cancer cells (11-13). The cucurbitacin family comprises a group of triterpenoid compounds originally isolated from the plants of the Cucurbitaceae family. Recently, these anticancer compounds have been observed in many plant families, including Cruciferae, Cucurbitaceae and Scrophulariaceae, and have been used as traditional or folk medicines for centuries in China, India, Brazil and Peru $(13,14)$. Previous studies have demonstrated that cucurbitacin I inhibits the JAK/STAT3 pathway in a number of cancer cell lines and in vivo tumor models $(11,15)$. Upon inhibition of STAT3-dependent gene transcription, cucurbitacin I elicits antiproliferative effects in glioma, lung and breast cancer cells with activated STAT3 $(13,16)$.

Limited research has been carried out concerning the effect of cucurbitacin I on colon cancer. A recently published study demonstrated that cucurbitacin I induced cell death and G2/M phase cell cycle arrest in SW480 cells (17). Our results in the present study confirmed this effect of cucurbitacin I on colon cancer cells. However, how cucurbitacin I influences colon cancer cell migration and invasion is still elusive. In the present study, we investigated the effect of cucurbitacin I on colon cancer cell migration and invasion, and whether cucurbitacin I enhances the chemosensitivity of the colon cancer cells. Furthermore, we also investigated the molecular mechanisms of cucurbitacin I function.

\section{Materials and methods}

Materials and cell culture. Cucurbitacin I was purchased from Calbiochem (Jersey City, NJ, USA) and dissolved in dimethyl sulfoxide (DMSO) at the concentration of $10 \mathrm{mM}$. The antibodies used in the study included: anti-phospho-STAT3-Tyr705 (Cell Signaling Technology, Danvers, MA, USA), anti-MMP-9 (Abcam, Cambridge, MA, USA) and anti-actin (Santa Cruz Biotechnology, Dallas, TX, USA). Secondary antibodies were purchased from Jackson ImmunoResearch (Baltimore, MD, USA). 

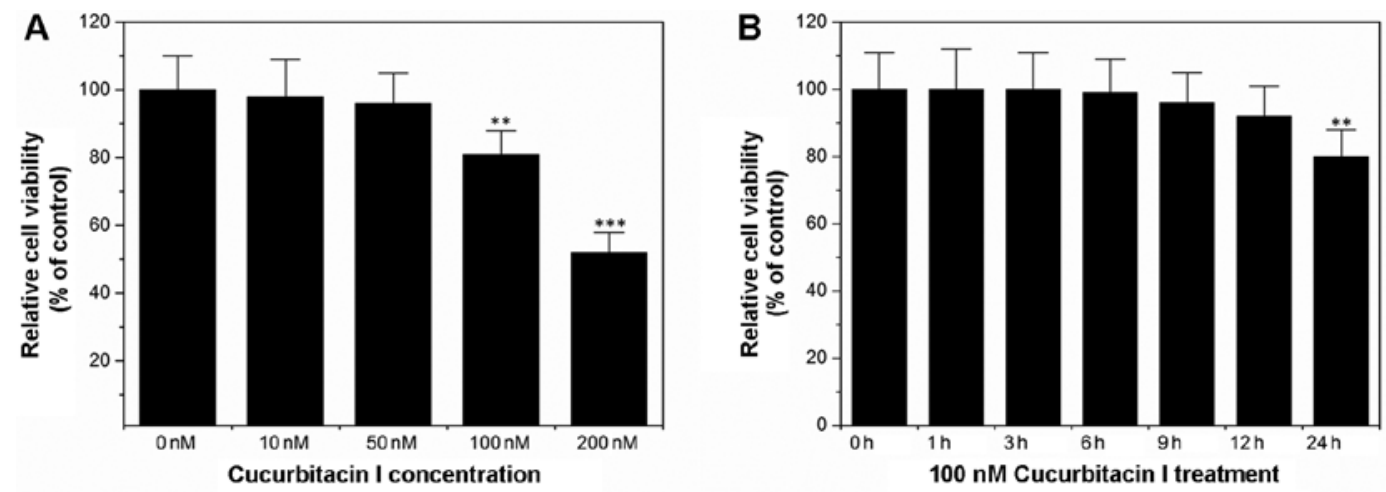

Figure 1. Cucurbitacin I induces colon cancer cell death. The COLO205 cells were treated with different doses of cucurbitacin I for (A) $24 \mathrm{~h}$ or with (B) $100 \mathrm{nM}$ cucurbitacin I for different times. The cell viability was determined by the CCK- 8 Kit. The cell viability was presented as a percentage of the control. ${ }^{* *} \mathrm{P}<0.05$ and ${ }^{* * *} \mathrm{P}<0.01$.

The COLO205 colon cancer cell line was purchased from the American Type Culture Collection (ATCC; Manassas, VA, USA) and cultured in Dulbecco's modified Eagle's medium. All media were supplemented with $10 \%$ fetal bovine serum (FBS) (Invitrogen, Madison, WI, USA), and $100 \mathrm{U} / \mathrm{ml}$ penicillin and $100 \mu \mathrm{g} / \mathrm{ml}$ streptomycin (Sigma, St. Louis, MO, USA).

Cell viability assays. Cell viability was measured by the CCK-8 Kit (Dojindo, Kumamoto, Japan). Briefly, cells were plated at the density of $1 \times 10^{4}$ cells/well in a 94-well plate. The following day, the cells were incubated with either DMSO or increasing concentrations of cucurbitacin I for $24 \mathrm{~h}$. The 1/10 volume of CCK- 8 solution of cultured medium was added to the cells. Then, the cells were further incubated at $37^{\circ} \mathrm{C}$ for $2 \mathrm{~h}$. The optical density (OD) at $450 \mathrm{~nm}$ was measured by using a VICTOR ${ }^{\mathrm{TM}} \mathrm{X}$ plate reader (PerkinElmer, Waltham, MA, USA). The percentage of cell viability was calculated as $\mathrm{OD}_{\text {drug }} / \mathrm{OD}_{\text {control }}$ x $100 \%$.

Cell migration and invasion assays. The in vitro migration assays were performed as previously described with some modifications $(18,19)$ using Transwells $(8-\mu \mathrm{m}$ pore size; $\mathrm{BD}$ Corporation, Franklin Lakes, NJ, USA). The COLO205 cells were added to the upper inserts of the chamber (200 $\mu 1$ serumfree medium containing $2 \times 10^{4}$ cells), and $600 \mu \mathrm{l}$ medium with $1 \%$ FBS was added to the lower well. After $6 \mathrm{~h}$ of incubation, the cells were removed from the upper surface of the filter with a cotton swab and the cells that migrated through the inserts were fixed with methanol and stained with crystal violet. The migrated cells were counted under a microscope (TS100; Nikon, Tokyo, Japan) and the migration ability of the control group was set as $100 \%$. The migration ability of the treated group was calculated as the migrated cell number of the drug-treated group/the migrated cell number of the control group x $100 \%$. Each experiment was performed in triplicate and this experiment was repeated three times.

Cell invasion assays were performed as the migration assays except that the Transwells used in the invasion assays were Matrigel-coated, while in the migration assays the Transwells remained uncoated.

Western blotting. The cell lysates were prepared as previously described (20). After DMSO or cucurbitacin I treatment for $24 \mathrm{~h}$, the cells were washed with ice-cold phosphate-buffered saline (PBS) three times and lysed in RIPA lysis buffer supplemented with a proteinase inhibitor (both from Beyotime, Nanjing, China) and $1 \mathrm{mM}$ phenylmethylsulfonyl fluoride (PMSF). Then, the cell lysates were harvested. Fifty grams of whole cell lysates was electrophoresed by sodium dodecyl sulfate-polyacrylamide gel electrophoresis (SDS-PAGE), electro-transferred to polyvinylidene fluoride (PVDF) membranes and probed with an appropriate primary antibody. Then the blots were next probed with appropriate horseradish peroxidase (HRP)-conjugated secondary antibodies (Santa Cruz Biotechnology, Santa Cruz, CA, USA) and were visualized by an enhanced chemiluminescence assay kit (ECL kit; Applygen Technology, Beijing, China). Membranes were also probed with an anti-actin antibody to monitor the sampling difference.

Statistical analysis. Statistical analysis was performed using SPSS 17.0 software (SPSS, Inc., Chicago, IL, USA). Data are presented as mean \pm standard deviation (SD) and were analyzed by the Student's t-test. A difference was considered to be statistically significant at $\mathrm{P}<0.05$.

\section{Results}

Cucurbitacin I suppresses colon cancer cell proliferation, migration and invasion in vitro. As shown in Fig. 1A, cucurbitacin I inhibited colon cancer cell COLO205 proliferation in a dose-dependent manner. These results confirmed a previous report by other researchers (17). However, to date it is not clear how cucurbitacin I affects colon cancer cell migration and invasion. In order to answer this question, Transwell assays were performed. Since Transwell assays take $\sim 6 \mathrm{~h}$, we firstly determined the cell viability after treatment with $100 \mathrm{nM}$ cucurbitacin I for $6 \mathrm{~h}$. As indicated in Fig. 1B, treatment with $100 \mathrm{nM}$ cucurbitacin I for $6 \mathrm{~h}$ had no statistically significant influence on the cell viability as compared with the control. Thus, the parameters of $100 \mathrm{nM}$ cucurbitacin I and the $6 \mathrm{~h}$ treatment were chosen for the Transwell assays. As illustrated in Fig. 2, $100 \mathrm{nM}$ cucurbitacin I treatment for $6 \mathrm{~h}$ reduced COLO205 colon cancer cell migration to $~ 50 \%$ as compared with the control $(\mathrm{P}<0.001)$. Cell invasion assays showed that $100 \mathrm{nM}$ cucurbitacin I also inhibited COLO205 colon cancer cell invasion in vitro (Fig. 3). 

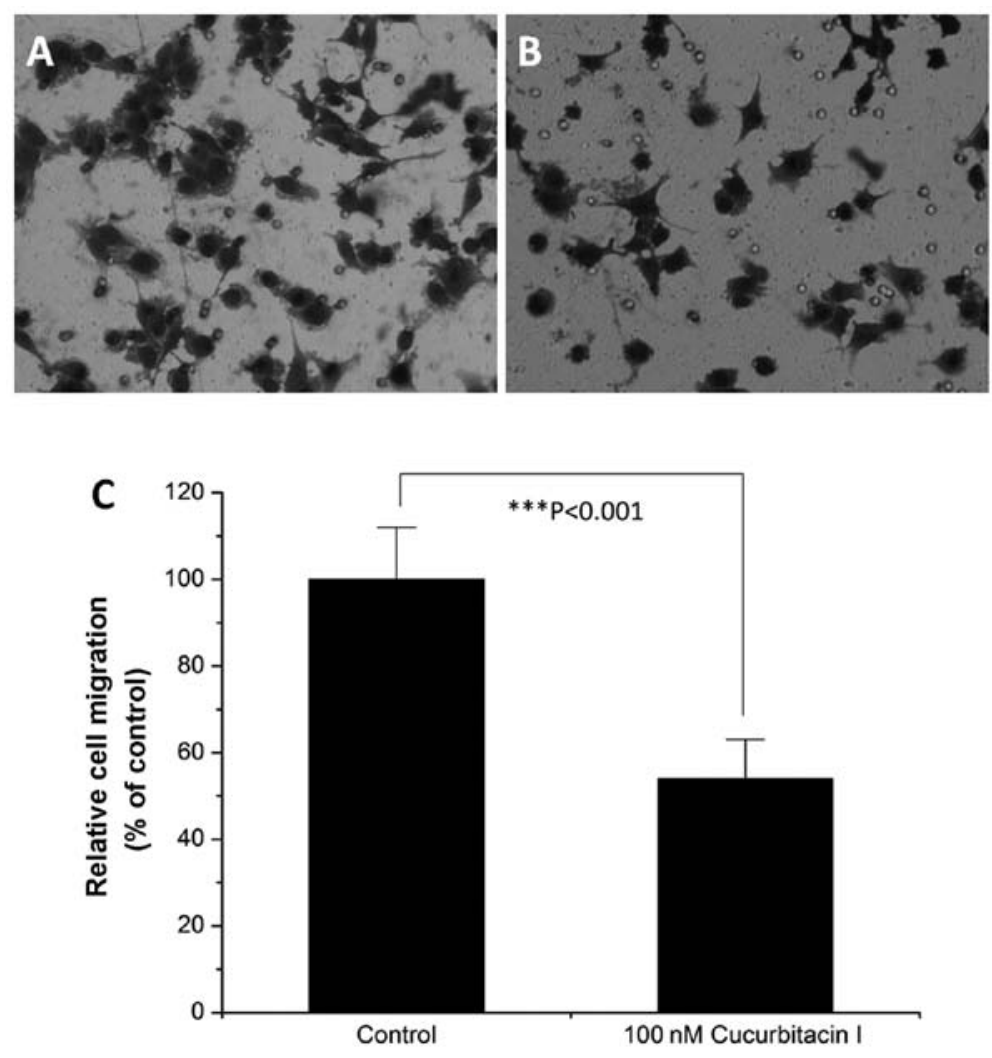

Figure 2. Cucurbitacin I inhibits colon cancer cell migration. (A) Control; (B) $100 \mathrm{nM}$ cucurbitacin I treatment. The COLO205 cells were seeded onto the upper Transwell inserts of the chamber without Matrigel at the same cell density of $2 \times 10^{4}$ cells. Six hours later, the cells that invaded through the inserts were stained and counted under a light microscope. (C) Finally, data were calculated and cell migration was presented as a percentage of the control. Similar results were obtained from three independent experiments.
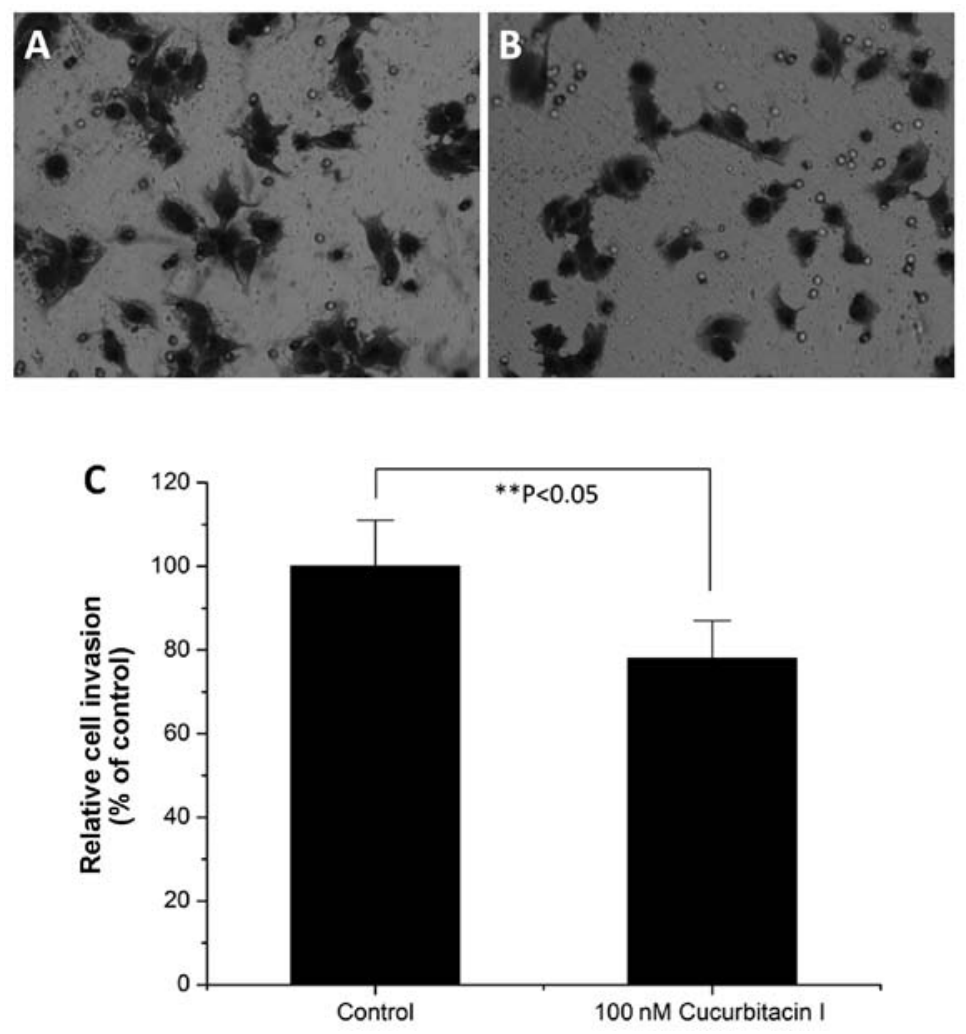

Figure 3. Cucurbitacin I suppresses colon cancer cell invasion. (A) Control; (B) $100 \mathrm{nM}$ cucurbitacin I treatment. The COLO205 cells were seeded onto the upper Transwell inserts of the Matrigel chamber at the same cell density of $2 \times 10^{4}$ cells. Six hours later, the cells that invaded through the Matrigel-coated inserts were stained and counted under a light microscope. (C) Finally, data were calculated and cell invasion was presented as a percentage of the control. Similar results were obtained from three independent experiments. 


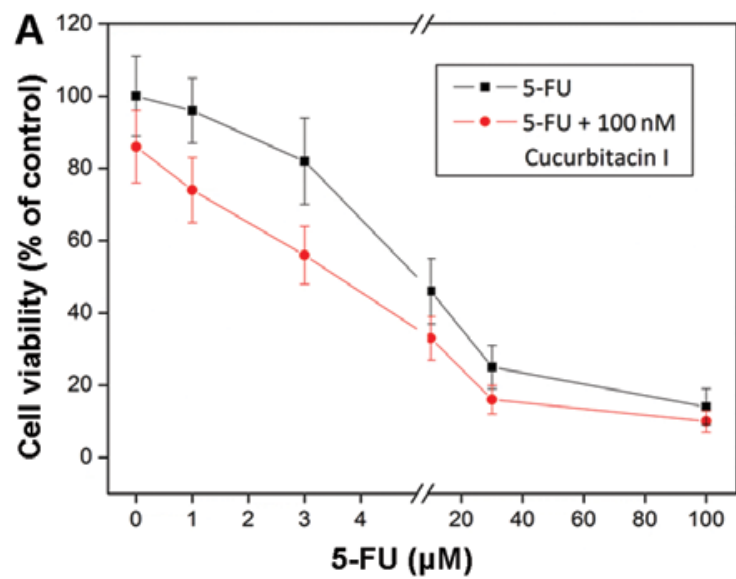

B

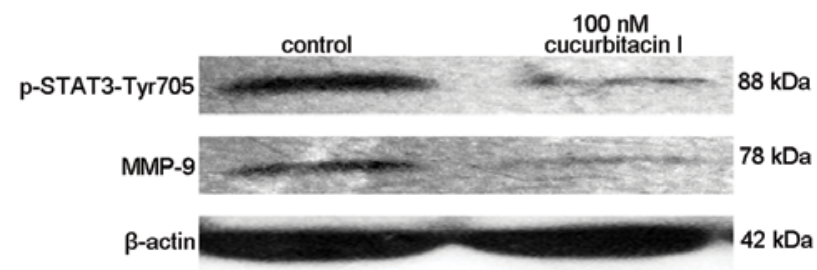

Figure 4. (A) Cucurbitacin I sensitizes colon cancer cell line COLO205 to 5-FU treatment. COLO205 colon cancer cells were seeded onto a 96-well plate at the same density ( $1 \times 10^{4}$ cells) and then treated with 5-FU alone or the combination of 5-FU and $100 \mathrm{nM}$ cucurbitacin. Twenty-four hours later, the cell viability was determined using the CCK-8 Kit. The cell viability was presented as a percentage of the control. This experiment was repeated three times with similar results. (B) Cucurbitacin I inhibited the expression of activated STAT3 in COLO205 cells. COLO205 cells were treated with $100 \mathrm{nM}$ cucurbitacin I for $24 \mathrm{~h}$ and then harvested for western blot analysis. The expression levels of phospho-STAT3-Tyr705 were compared between the control and cucurbitacin I-treated COLO205 cells. Actin is shown as a loading control. Similar results were obtained in three independent experiments.

Cucurbitacin I sensitizes colon cancer cell to chemotherapeutic agents. As confirmed above by our results and a number of previous reports $(11,13,17,21,22)$, cucurbitacin I inhibits tumor cell proliferation, migration and invasion, which makes cucurbitacin I a promising antitumor target (23). However, to date it is not known whether cucurbitacin I has a sensitizing effect on colon cancer cells to chemotherapy. In the present study, we combined the chemotherapy with the cucurbitacin I treatment. As shown in Fig. 4A, the cell death was further enhanced by the combination treatment of 5-fluorouracil (5-FU) and $100 \mathrm{nM}$ cucurbitacin I, which proved that cucurbitacin I sensitized the colon cancer cell line COLO205 to 5-FU treatment.

Cucurbitacin I suppresses STAT3 activation and decreases MMP-9 expression. Previous reports have shown that cucurbitacin I suppresses STAT3 activation in other cancer cell lines, such as breast cancer (24) and leukemia cells (25). In the present study, we determined the effect of cucurbitacin I on STAT3 in colon cancer cells. As indicated in Fig. 4B, $100 \mathrm{nM}$ cucurbitacin decreased the protein level of phospho-STAT3, which proved the inhibitory effect of cucurbitacin I on STAT3 activation.

MMP-9 is an important enzyme for tumor cell invasion (26). As cucurbitacin inhibited colon cancer cell invasion, we determined whether cucurbitacin I treatment could change the MMP-9 expression level. Western blotting results showed that the MMP-9 expression level was decreased by $100 \mathrm{nM}$ cucurbitacin treatment (Fig. 4B).

\section{Discussion}

At present, natural chemical compounds are important targets in anticancer research due to the drug resistance and toxic side-effects of current chemotherapy. Herbal medicine has attracted increased attention of medical scientists (27). Cucurbitacin I is a natural component extracted from plants of the Cucurbitaceae family, and exerts anticancer activities in glioblastoma, adenocarcinoma of the lung and breast cancer cells (11-13). The molecular mechanisms of cucurbitacin I function involve the inhibition of STAT3 activation $(13,24)$, and interference with actin dynamics $(28,29)$. A recently published study (17) demonstrated that in SW480 cells cucurbitacin I decreased the cell viability and cell proliferation by cleavage of caspase-3, - $7,-8$ and -9 and polyADP ribose polymerase and induced $\mathrm{G} 2 / \mathrm{M}$ cell cycle arrest by downregulation of the cell cycle proteins including cyclin B1 and A, CDK1 and CDC25C. In the present study, we confirmed the inhibitory effect of cucurbitacin I on colon cancer cell growth as previously reported (17). Our results further showed that cucurbitacin I inhibited colon cancer cell migration and invasion in vitro, and sensitized colon cancer cells to 5-FU treatment.

Transcription factor STAT3 has been implicated in the promotion of growth and progression of many human cancers including gastric cancers (30-37). STAT3 is both a cytoplasmic signaling molecule and a nuclear transcription factor, which belongs to the seven-member Stat gene family of transcription factors (38). STAT3 becomes active by phosphorylation of a specific tyrosine residue in the carboxy-terminal domain by a tyrosine kinase (pTyr705). After phosphorylation, STAT3 homodimerizes and translocates to the nucleus where it binds to specific STAT3 response elements of target gene promoters to regulate transcription (39). Transcription factor STAT3 is constitutively active in many human cancers $(30,40)$. In the present study, we firstly observed that in colon cancer cells cucurbitacin I suppressed phosphorylation of STAT3 (pTyr705). As STAT3 activation was involved in tumor metastasis (31), inhibition of cucurbitacin I on STAT3 activation could explain its inhibitory effect on colon cancer cell migration and invasion. Previous findings showed that, in medulloblastoma-derived cancer stem cells, cucurbitacin I enhanced chemoradiosensitivity by inhibiting STAT3 phosphorylation (40). In our results we observed that cucurbitacin I sensitized colon cancer cells to chemotherapy, which may 
be promoted by inhibiting STAT3 activation. Lastly, we also observed that cucurbitacin I decreased the MMP-9 expression which is an important enzyme for cell invasion (26).

In conclusion, the present study showed that cucurbitacin I exhibited inhibitory effects on colon cancer cell proliferation, migration and invasion, which may be accomplished by downregulating phosphorylation of STAT3 and MMP-9 expression. Our results also indicated that cucurbitacin I could sensitize colon cancer cells to chemotherapy.

\section{Acknowledgements}

The present study was supported by the Joint Program of the National Natural Science Foundation of China (NSFC), and the Science and Technology Agency of Henan Province (no. U1204818).

\section{References}

1. Siegel R, Naishadham D and Jemal A: Cancer statistics, 2012. CA Cancer J Clin 62: 10-29, 2012.

2. Cunningham D, Atkin W, Lenz HJ, et al: Colorectal cancer Lancet 375: 1030-1047, 2010.

3. Labianca R, Beretta GD, Kildani B, et al: Colon cancer. Crit Rev Oncol Hematol 74: 106-133, 2010.

4. Pihl E, Hughes ES, McDermott FT, Milne BJ and Price AB Disease-free survival and recurrence after resection of colorectal carcinoma. J Surg Oncol 16: 333-341, 1981.

5. Cresanta JL: Epidemiology of cancer in the United States. Prim Care 19: 419-441, 1992.

6. Greenwald P: Colon cancer overview. Cancer 70 (Suppl 5): S1206-S1215, 1992.

7. Zhang H, Qian Y, Liu Y, et al: Celastrus orbiculatus extract induces mitochondrial-mediated apoptosis in human hepatocellular carcinoma cells. J Tradit Chin Med 32: 621-626, 2012.

8. Liu J and Liu Y: Influence of Erbanxiao solution on inhibiting angiogenesis in stasis toxin stagnation of non-small cell lung cancer. J Tradit Chin Med 33: 303-306, 2013.

9. Kang J, Lee N, Ahn Y and Lee H: Study on improving blood flow with Korean red ginseng substances using digital infrared thermal imaging and Doppler sonography: randomized, double blind, placebo-controlled clinical trial with parallel design. J Tradit Chin Med 33: 39-45, 2013.

10. Surh YJ: Anti-tumor promoting potential of selected spice ingredients with antioxidative and anti-inflammatory activities: a short review. Food Chem Toxicol 40: 1091-1097, 2002.

11. Su Y, Li G, Zhang X, et al: JSI-124 inhibits glioblastoma multiforme cell proliferation through $\mathrm{G} 2 / \mathrm{M}$ cell cycle arrest and apoptosis augment. Cancer Biol Ther 7: 1243-1249, 2008.

12. van Kester MS, Out-Luiting JJ, von dem Borne PA, Willemze R, Tensen CP and Vermeer MH: Cucurbitacin I inhibits Stat3 and induces apoptosis in Sézary cells. J Invest Dermatol 128: $1691-1695,2008$

13. Blaskovich MA, Sun J, Cantor A, Turkson J, Jove R and Sebti SM: Discovery of JSI-124 (cucurbitacin I), a selective Janus kinase/ signal transducer and activator of transcription 3 signaling pathway inhibitor with potent antitumor activity against human and murine cancer cells in mice. Cancer Res 63: 1270-1279, 2003.

14. Chen JC, Chiu MH, Nie RL, Cordell GA and Qiu SX: Cucurbitacins and cucurbitane glycosides: structures and biological activities. Nat Prod Rep 22: 386-399, 2005.

15. Chen CL, Hsieh FC, Lieblein JC, et al: Stat 3 activation in human endometrial and cervical cancers. Br J Cancer 96 . 591-599, 2007.

16. Lo HW, Cao X, Zhu H and Ali-Osman F: Constitutively activated STAT3 frequently coexpresses with epidermal growth factor receptor in high-grade gliomas and targeting STAT3 sensitizes them to Iressa and alkylators. Clin Cancer Res 14: 6042-6054, 2008.

17. Kim HJ, Park JH and Kim JK: Cucurbitacin-I, a natural cellpermeable triterpenoid isolated from Cucurbitaceae, exerts potent anticancer effect in colon cancer. Chem Biol Interact 219: $1-8,2014$
18. Gu L, Feng J, Xu H, Luo M and Su D: Polyphyllin I inhibits proliferation and metastasis of ovarian cancer cell line HO-8910PM in vitro. J Tradit Chin Med 33: 325-333, 2013.

19. Luo XS, Wu XW and Gu Q: An experimental study of a modified Dahuang Zhechong pill on the - angiogenesis of RF/6A cells in vitro. J Tradit Chin Med 32: 75-81, 2012.

20. Lui VW, Boehm AL, Koppikar P, et al: Antiproliferative mechanisms of a transcription factor decoy targeting signal transducer and activator of transcription (STAT) 3: the role of STAT1. Mol Pharmacol 71: 1435-1443, 2007.

21. Hsu HS, Huang PI, Chang YL, et al: Cucurbitacin I inhibits tumorigenic ability and enhances radiochemosensitivity in nonsmall cell lung cancer-derived CD133-positive cells. Cancer 117: 2970-2985, 2011.

22. Chen YW, Chen KH, Huang PI, et al: Cucurbitacin I suppressed stem-like property and enhanced radiation-induced apoptosis in head and neck squamous carcinoma-derived CD $44^{+} \mathrm{ALDH} 1^{+}$ cells. Mol Cancer Ther 9: 2879-2892, 2010.

23. Alghasham AA: Cucurbitacins - a promising target for cancer therapy. Int J Health Sci 7: 77-89, 2013.

24. Ren Y, Yu K, Sun S, et al: JSI124 inhibits breast cancer cell growth by suppressing the function of B cells via the downregulation of signal transducer and activator of transcription 3. Oncol Lett 8: 928-932, 2014.

25. Ishdorj G, Johnston JB and Gibson SB: Inhibition of constitutive activation of STAT3 by curcurbitacin-I (JSI-124) sensitized human B-leukemia cells to apoptosis. Mol Cancer Ther 9: 3302-3314, 2010

26. Björklund $M$ and Koivunen E: Gelatinase-mediated migration and invasion of cancer cells. Biochim Biophys Acta 1755: 37-69, 2005.

27. Li X, Yang G, Li X, et al: Traditional Chinese medicine in cancer care: a review of controlled clinical studies published in Chinese. PLoS One 8: e60338, 2013.

28. Knecht DA, LaFleur RA, Kahsai AW, Argueta CE, Beshir AB and Fenteany G: Cucurbitacin I inhibits cell motility by indirectly interfering with actin dynamics. PLoS One 5: e14039, 2010.

29. Maloney KN, Fujita M, Eggert US, et al: Actin-aggregating cucurbitacins from Physocarpus capitatus. J Nat Prod 71: 1927-1929, 2008.

30. Abdulghani J, Gu L, Dagvadorj A, et al: Stat3 promotes metastatic progression of prostate cancer. Am J Pathol 172: 1717-1728, 2008.

31. Deng JY, Sun D, Liu XY, Pan Y and Liang H: STAT-3 correlates with lymph node metastasis and cell survival in gastric cancer. World J Gastroenterol 16: 5380-5387, 2010.

32. Dauer DJ, Ferraro B, Song L, et al: Stat 3 regulates genes common to both wound healing and cancer. Oncogene 24: 3397-3408, 2005.

33. Xie TX, Huang FJ, Aldape KD, et al: Activation of stat 3 in human melanoma promotes brain metastasis. Cancer Res 66: 3188-3196, 2006.

34. Li WC, Ye SL, Sun RX, et al: Inhibition of growth and metastasis of human hepatocellular carcinoma by antisense oligonucleotide targeting signal transducer and activator of transcription 3. Clin Cancer Res 12: 7140-7148, 2006.

35. Silver DL, Naora H, Liu J, Cheng W and Montell DJ: Activated signal transducer and activator of transcription (STAT) 3: localization in focal adhesions and function in ovarian cancer cell motility. Cancer Res 64: 3550-3558, 2004.

36. Horiguchi A, Oya M, Shimada T, Uchida A, Marumo K and Murai M: Activation of signal transducer and activator of transcription 3 in renal cell carcinoma: a study of incidence and its association with pathological features and clinical outcome. J Urol 168: 762-765, 2002.

37. Kusaba T, Nakayama T, Yamazumi K, et al: Expression of p-STAT3 in human colorectal adenocarcinoma and adenoma; correlation with clinicopathological factors. J Clin Pathol 58: 833-838, 2005

38. Ihle JN: The Stat family in cytokine signaling. Curr Opin Cell Biol 13: 211-217, 2001.

39. Levy DE and Darnell JE Jr: Stats: transcriptional control and biological impact. Nat Rev Mol Cell Biol 3: 651-662, 2002.

40. Chang CJ, Chiang CH, Song WS, et al: Inhibition of phosphorylated STAT3 by cucurbitacin I enhances chemoradiosensitivity in medulloblastoma-derived cancer stem cells. Childs Nerv Syst 28: 363-373, 2012. 